\title{
Intraoral schwannoma in a seven-year-old boy - a case report
}

\author{
Santosh Kumar Swain, Rohit Agrawala \\ Department of Otorhinolaryngology, IMS and SUM Hospital, Siksha "O" Anusandhan University, K8, Kalinganagar, \\ Bhubaneswar, Odisha, India
}

\section{ABSTRACT}

Schwannoma is a benign neoplasm which arises from the Schwann cells of the peripheral nerve sheath. It is a common neoplasm in the head and neck region; however, intraoral location is extremely rare. It occurs infrequently in the oral cavity of children. Here we report a case lingual schwannoma in a seven-year-old boy who presented with a small, smooth, firm, non-tender mass at the right border of the tongue. Histopathological and immunohistochemical examination confirmed the diagnosis of lingual schwannoma. These tumours are usually benign in nature and have excellent prognosis with low potential for malignant transformation. Surgical excision of the tumour is the primary treatment of choice in cases of lingual schwannoma. The transoral route is considered as the most commonly used approach for tongue schwannoma.

\section{KEY WORDS:}

schwannoma, tongue, oral cavity, MRI.

\section{INTRODUCTION}

Schwannoma is a benign neoplasm that arises from Schwann cells of the peripheral nerve sheath. It is a slow-growing, solitary, and encapsulated tumour. It is a common neoplasm in the head and neck region, and around $25-45 \%$ of all schwannomas are found in that region. However, its location in the oral cavity (approximately 1\%) is extremely uncommon [1]. It can arise from any peripheral, cranial or autonomic nerves that contain Schwann cells - the cells which form the myelin sheath over the nerve fibres. The standard treatment approach is transoral resection of the tumour. The recurrence of this tumour is very rare, and malignant transformation is also rare [2]. Schwannoma was first documented by Verocay in 1910, who coined this benign neurogenic tumour as neuriloma [3]. This tumour may arise at any age, but the peak incidence is between the third and sixth decades of life, without any sex predilection [2]. Here, we present a case of schwannoma at the lateral border of the tongue in a paediatric patient.

\section{CASE REPORT}

A seven-year-old boy presented a slow growing and painless mass on the right border of the tongue for one year. The patient had no other complaints except a swelling in the tongue. No significant personal or family medical history was reported. Examination of the oral cavity revealed the presence of a firm and non-tender mass with size $1 \mathrm{~cm} \times 1.5 \mathrm{~cm}$ at the right lateral border of the tongue (Fig. 1). He had no cervical node enlargement and other clinical examinations within normal limits. A magnetic resonance imaging (MRI) was advised assess the lesion, which showed an ovulated nodular mass at the right border of the tongue, well circumscribed, homogeneously

\section{ADDRESS FOR CORRESPONDENCE:}

Santosh Kumar Swain, Department of Otorhinolaryngology, IMS and SUM Hospital, Siksha "O" Anusandhan

University, K8, Kalinganagar, 751003 Bhubaneswar, Odisha, India, ORCID: 0000-0001-7933-4414,

e-mail: santoshvoltaire@yahoo.co.in 


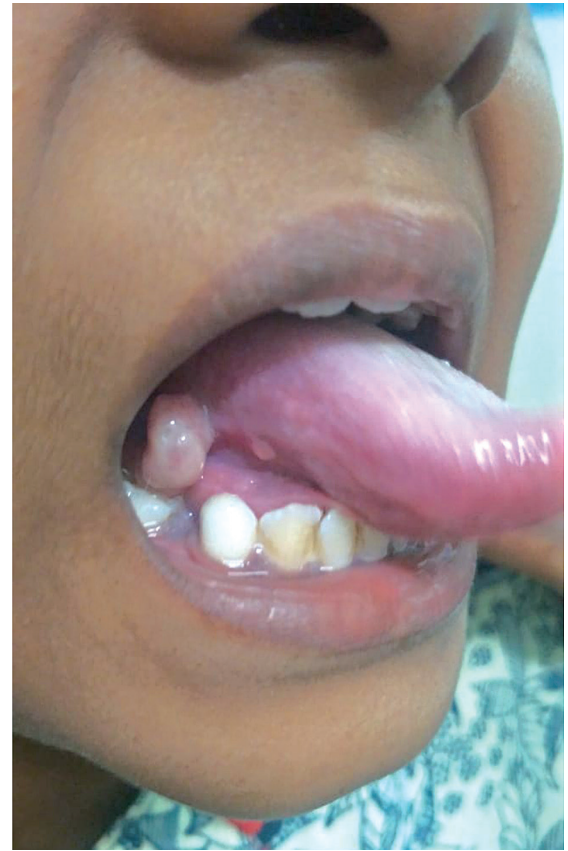

FIGURE1. Firm and non-tender mass (schwannoma) with size $1 \mathrm{~cm} \times 1.5 \mathrm{~cm}$ size at the right lateral border of the tongue

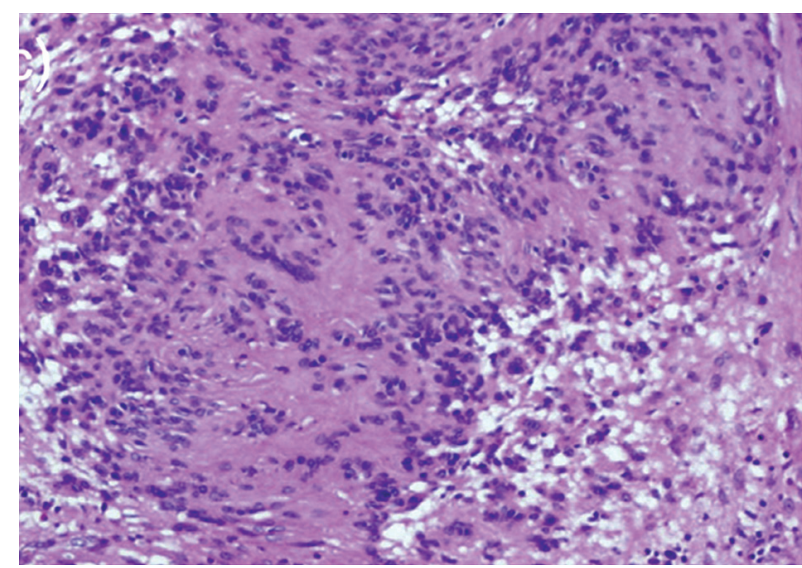

FIGURE 3. Schwannoma of the tongue showing spindle cells arranged in palisading pattern and forming Verocay bodies (Antoni $A$ area) (H\&E stain, $100 \times)$

isointense to the muscle on T1W1, and homogeneously hyperintense on $\mathrm{T} 2 \mathrm{~W} 1$, revealing homogeneous hypervascular enhancement in the contrast dynamic study (Fig. 2). For confirmation of the diagnosis, the mass was excised under local anaesthesia and sent for histopathological examination. The histopathological examination and immunohistochemical examination confirmed the diagnosis of schwannoma. Gross examination of the excised mass showed a mucosa-covered, yellow to white, polypoidal, nodular appearance. A cut section of the mass showed smooth, pale white, glistening neoplasm with streaks of haemorrhage. Histopathological examination of this tumour showed a circumscribed, well-defined lesion covered by a thin lining of mucosa. The tumour consisted of alternate hypocellular and hypercellular areas made up of spindle shaped Schwann cells. The hypercellular (Anto-

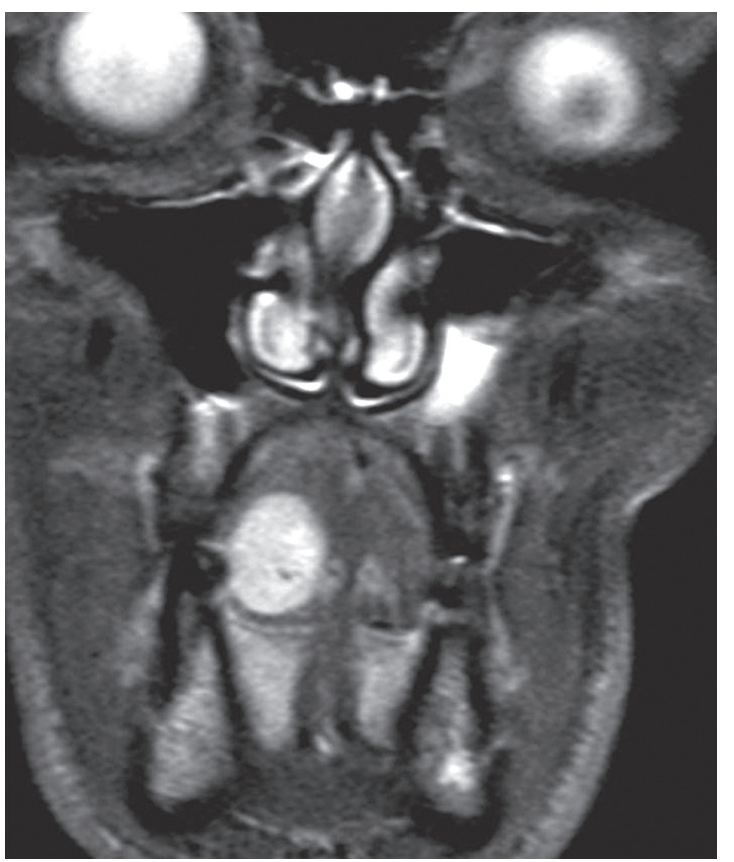

FIGURE 2. MRI of the tongue revealed the presence of a well-circumscribed nodule on the right anterior tongue, homogeneously and homo-geneously hyperintense on T2WI image

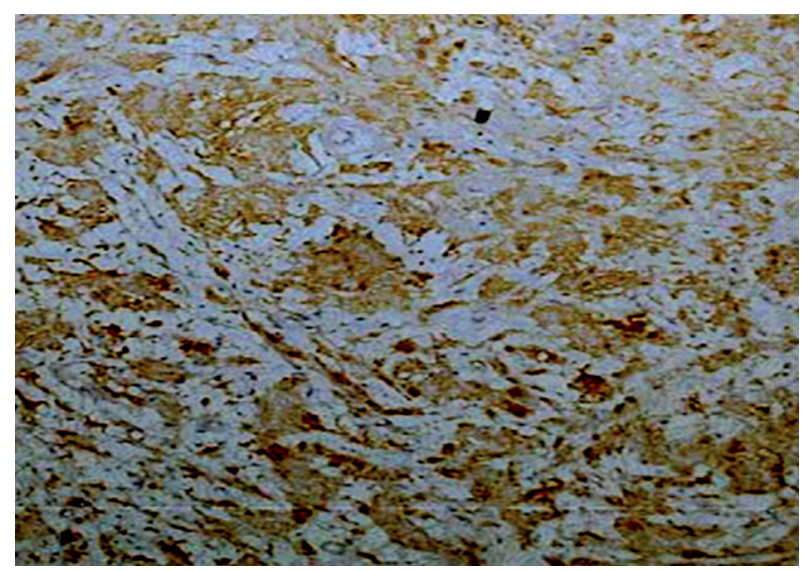

FIGURE 4. Schwannoma at the tongue showing strong S-100 reactivity $(200 \times)$

ni A) areas showed cellular interlacing eosinophilic areas characteristic of Verocay bodies (Fig. 3). The hypocellular areas (Antoni B) showed myxoid change. The immunochemistry of this tumour showed strong S100 positivity in the spindle tumour cells (Fig. 4). Cytokeratin and SMA were negative in these neoplastic cells. Ki67 staining revealed low proliferative index. So, it was diagnosed as a benign schwannoma arising from the right lateral border of the tongue. At six months and then at one year after surgery the child did not show any recurrence.

\section{DISCUSSION}

Peripheral nerve sheath tumours are extremely rare in the oral cavity. These tumours include schwannoma (neurilemmoma or neurinomas), neurofibromas, and 
traumatic neuromas. Schwannomas are benign and encapsulated tumours that arise from the spinal roots, cervical nerves, sympathetic nerves, vagus, peroneal, and ulnar nerves. Neurofibromas are composed of Schwann cells, neuritis, and fibroblasts within a collagenous matrix [4]. Schwannomas can originate from all the cranial nerves except the first and second (CN I and CNII), whereas CN VIII is the most commonly affected cranial nerve. Other sites include spinal nerve roots, flexor surfaces of lower and upper extremities, and tongue [5]. Although the majority of schwannomas are solitary, there is an association with neurofibromatosis type 2 [1]. Extracranially, approximately $25 \%$ of all the schwannomas are found in the head and neck area, but only $1 \%$ are seen in the intraoral region [6]. These intraoral tumours have predilection for the tongue followed by the palate, buccal mucosa, lips, and gingival. These tumours are often asymptomatic unless they grow to an appreciable size. Schwannomas are often seen in the age groups of 20 to 50 years, and they affect both males and females in equal number [7]. Schwannomas of the tongue usually arise from the hypoglossal nerve. Typically, this tumour is asymptomatic and slow growing in nature, presenting for several years before diagnosis. These tumours become symptomatic by virtue of their large size and their application of pressure to the affected nerve [8]. They are usually slow growing with a smooth surface, and they are usually asymptomatic. The tumour is often single, firm, circumscribed, painless, and variable in size. Sometimes ulceration is seen over the mass, which is due to trauma [9]. Although this tumour is usually solitary, sometimes these are multiple or are seen in the setting of von-Recklinghausen's neurofibromatosis, which almost never undergo malignant transformation. Any part of the tongue can be affected by schwannoma. Robert et al. and Mevio et al. documented schwannoma at the ventral surface of the tongue $[7,10]$. Other studies documented schwannoma at the base of the tongue and the tip of the tongue [7]. In our case, the schwannoma was confined to the lateral border of the tongue. The differential diagnoses of the lingual schwannoma include soft tissue tumours, malignancy, and salivary gland tumours. The histological differential diagnoses include spindle cell lesions, squamous cell carcinoma, smooth muscle tumours, fibroblastic neoplasms, lipoma, and rarely salivary gland neoplasms [11].

Imaging is an integral part of the assessment for tongue lesions, and so a systematic approach is required. The imaging choice of lingual schwannomas is MRI. MRI provides precise localisation of the tumour and better visualisation of the surrounding relations to other structures as well as exact measurement of the size of the tumour. MRI shows a well circumscribed tumour, homogenously isointense muscle on T1W1, and homogeneously hyperintense on T2W1. It also shows homogenous enhancement after contrast administration. Contrast CT scan of the schwannoma in the tongue reveals heterogenous enhancement [12]. It can also show cystic and fatty degeneration in the tongue. The histopathological diagnosis of the schwannoma with the presence of an alternate pattern of Antoni A and B areas, nuclear palisading, Verocay bodies, and whirling of cells is often unequivocal. Histologically, tumours mimicking neurilemmomas are meningioma, leiomyoma/leiomyosarcoma, palisaded fibroblastoma, and pleomorphic hyalinising angiectatic tumour of the soft tissue [8]. The genetic study of the patient can be undertaken in the case of atypical age especially for gene NF1. The genetic study is helpful from a cognitive point of view.

Complete surgical resection of the tumour is the standard treatment for schwannoma, including of the tongue [13]. The surgery for schwannoma in the oral cavity, either an intraoral approach or extra-oral approach depending on the size and location of the tumour. In our case, the tumour was located at the lateral border of the tongue which required surgical excision via transoral approach. In addition, preoperative assessment of the airway is required if the tumour is very large in size. In the case of surgery under general anaesthesia, nasotracheal intubation is preferable in intraoral schwannoma for better assessment and removal of the tumour. Recurrence of the lingual schwannoma has never been reported after complete resection [14]. Although malignant transformation is extremely rare, long-term follow-up is always required [15].

\section{CONCLUSIONS}

Schwannoma at the lateral border of the tongue is extremely rare in daily clinical practice. It can be considered as a differential diagnosis in benign lesions of the tongue. The final diagnosis is confirmed after histopathological examination and immunohistochemical analysis. The treatment of choice is complete excision of this tumour and it has excellent prognosis. These tumours usually do not show recurrence after complete surgical excision.

\section{DISCLOSURE}

The authors declare no conflict of interest.

\section{REFERENCES}

1. Cohen M, Wang B. Schwannoma of the tongue: two case reports and review literature. Eur Arch Otorhinolaryngol 2009; 266: 1823-1829.

2. Kavčič J, Božič M. Schwannoma of the tongue. BMJ Case Reports 2016; 2016: bcr2016215799.

3. Lollar WK, Pollak N, Liess DB, et al. Schwannoma of the hard palate. Am J Otolaryngol 2010; 31: 139-140.

4. Butler RT, Patel RM, McHugh JB. Head and neck schwannomas: 20-yearexperience of a single institution excluding cutaneous and acoustic sites. Head Neck Pathol 2016; 10: 286-291.

5. Stull MA, Moser Jr RP, Kransdorf MJ, et al. Magnetic resonance appearance of peripheral nerve sheath tumors. Skeletal Radiol 1991; 20: 9-14. 
6. Leu YS, Chang KC. Extracranial head and neck schwannomas: a review of 8 years' experience. Acta Oto Laryngol 2002; 122: 435-437.

7. Robert P, Dale AB, Augusto P, Joseph H. Schwannoma of the tongue: report of 2 cases. J Oral Maxillofac Surg 2001; 59: 802-804.

8. Enzinger FM, Weiss SW. Benign tumors of peripheral nerves. In: Soft tissue tumors. 4th ed. Enzinger FM, Weiss SW (eds.). Mosby, St. Louis 1995: 821-888.

9. Bansal R, Trivedi P, Patel S. Schwannoma of the tongue. Oral Oncol Extra 2005; 41: 15-17.

10. Mevio E, Gorini E, Lenzi A, Migliorini L. Schwannoma of the tongue: one case report. Rev Laryngol Otlol Rhinol 2002; 123: 259261.

11. Martins DM, Jesus LA, Fernandes SPK, et al. Intraoral schwannoma: case report and literature review. Indian J Dent Res 2009; 20: 121-125.

12. Fang WS, Wiggins $3^{\text {rd }} \mathrm{RH}$, Illner A, et al. Primary lesions of the root of the tongue. Radiographics 2011; 31: 1907-1922.

13. Rathore AS, Srivastava D, Narwal N, Shetty DC. Neurilemmoma of Retromolar Region in the oral cavity. Case Rep Dent 2015; 2015: 320830.

14. Lira RB, Gonçalves Filho J, Carvalho GB, et al. Lingual schwannoma: case report and review of the literature. Acta Otorhinolaryngol Ital 2013; 33: 137-140.

15. Kandasamy S, Nathan RS, John RR. Neurilemmoma of maxillary alveolus: a rare case report and review of literature. J Pharm Bioallied Sci 2017; 9: 285-288. 